\title{
Wrist Pulse Classification System for Healthy and Unhealthy Subjects
}

\author{
Kruti Parikh \\ Student, \\ G. H. Patel College of Engineering \\ \& Technology \\ V. V. Nagar.
}

\author{
Bhaskar Thakker, PhD \\ Head of Department, \\ Electronics \& Communication Dept. \\ G.H. Patel College of Engineering \\ \& Technology, V. V. Nagar
}

\begin{abstract}
According to Ayurveda, human health status can be measured by using three fingers as 'VATA', 'PITTA' and 'KAPHA' called Tridosha. This is very effective method to know the status of human body. But for this type of diagnosis practitioner requires long term training. Also the diagnoses from practitioner often deviate greatly due to their subjective experience. Thus, it is highly required to design such a system which diagnoses human health status objectively not subjectively.

In this paper, design of an embedded system, which classifies the health status, is discussed. This system first convert time domain signal into frequency domain using Fast Fourier Transform (FFT). Using Band Energy Ratio (BER) feature vector is retrieved from frequency domain signal. This feature vector is applied to the linear as well as quadratic classifier. This will classify the human health in a group of two as healthy or unhealthy.
\end{abstract}

\section{General terms}

Biomedical, Signal Processing, Classification

\section{Keywords}

Ayurveda, Fast Fourier Transform (FFT), Band Energy Ratio (BER), Classifier.

\section{INTRODUCTION}

In ancient India and China, practitioner following radial pulse analysis for the health diagnosis, uses three fingers for palpation. The key aspiration behind using radial pulse is, one can easily get the pulse as it is close to the $\operatorname{skin}^{[1]}$ and also it contains much more information about the human health status of the human body. The foremost purpose of pulse diagnosis is to obtain useful information about what goes on inside the body, what has caused disease, what might be done to rectify the problem, and what are the chances of diagnosis.

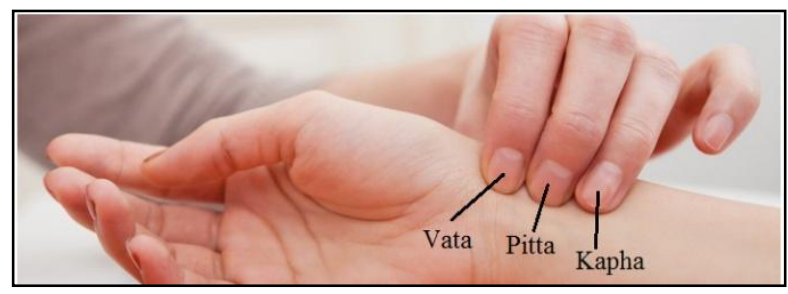

Figure 1: Traditional method of diagnosis based on wrist pulse ${ }^{[2]}$.

The chief limitation of pulse diagnosis lies due to its nature of usage where the practitioner finds it difficult to express the characteristics of pulse signals. Due to this subjective nature of the approach, it is very difficult to characterise the signals for the knowledge transfer. This is the reason for which this type of diagnosis depends immensely on the experience and subjective sensing of the practitioner.

The use of modern technology helps in minimizing this subjective nature by acquiring and characterizing the wrist pulse signals for time and frequency domain parameters ${ }^{[3]}$.

The main aim of this work is to classify the human health status in a group of two either healthy or unhealthy. Acquired wrist pulse for the healthy and unhealthy subjects are as shown in fig. 2 and fig. 3 respectively.

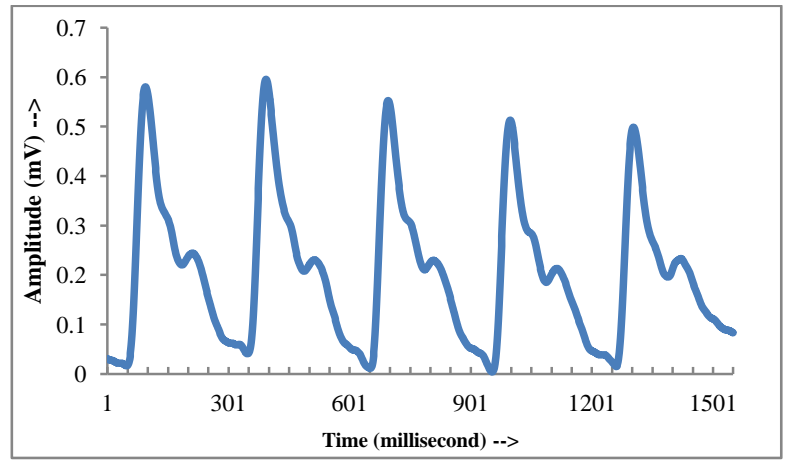

Figure 2: An example of healthy subject.

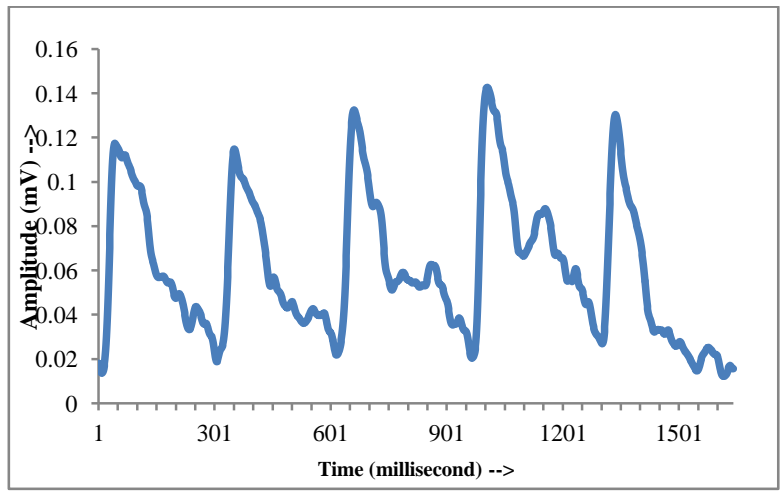

Figure 3: An example of unhealthy subject.

For the classification of human health status, wrist pulse is to be acquired from the human body, normalize the same and then the normalized pulse is given to the controller for feature extraction and further classification. Advance microcontroller stm32f4 cortex M4 is used for the classification.

In this work, Fast Fourier Transform (FFT) is used for frequency domain feature extraction from the time domain signal. From this frequency domain features, Band Energy Ratio (BER) is going to be calculated. This feature vector is 
given to the linear as well as quadratic classifier for further classification.

\section{RADIAL PULSE ACQUISITION AND PRE-PROCESSING}

Wrist pulse is mainly derived from the disciplinary beats of heart. When the heart beats periodically between systole and diastole, the blood ejects from left ventricle pounds of the aorta valve and wall ${ }^{[3]}$. It generates a sort of vibration in a form of waveform which transmits from the root of the aorta to any other arteries, is called forward wave or percussion wave. If the forward wave is affected by surrounding arterial branches, it reforms to the waveform in the inverse direction that is, Reflection Wave or tidal and dicrotic wave. Combination of both the waveforms generates a pulse as shown in fig. 4.

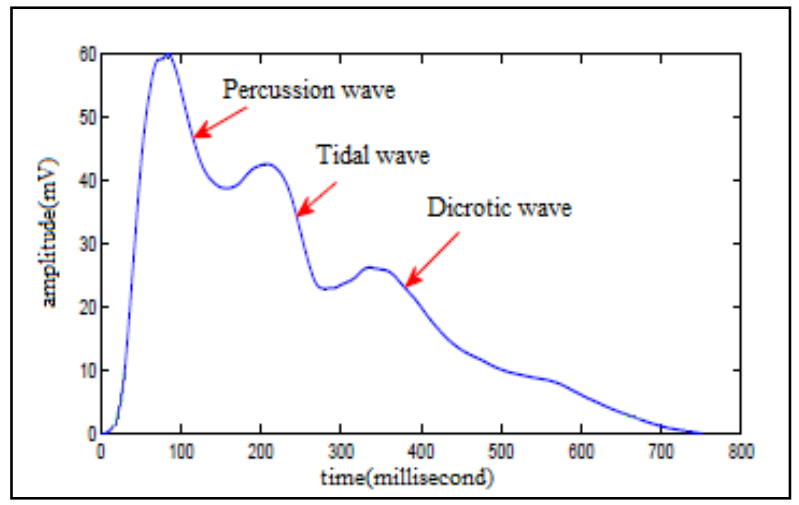

Figure 4: Single period time domain signal ${ }^{[4]}$.

Pulse can be acquired from the artery of the wrist using pressure sensor MPXM2053D. After acquisition, acquired pulse is interfaced with amplifier circuitry to appropriately amplify it and also interfaced with some signal conditioning circuitry to reduce the noise present during the acquisition. Also acquired pulse has some power line pick-ups. To reduce this type of noise notch filter is used. Wrist pulse is very low frequency signal, therefore signal is passed through the low pass filter to remove the high frequency component from the signal $^{[1]}$.

\section{COMPONENT SELECTION}

Classification system design comprises stm32f4 cortex M4 series advance microcontroller for feature extraction and classification purpose. Brief description on stm32f4 microcontroller is discussed in this section.

\subsection{STM32F429 Discovery board:}

The choice of this board is made according to the features supported by it to suffice system requirements. It is an ARM 32-bit Cortex-M4 CPU with FPU. Main advantage of this board is, it is having FPU so one can get the better accuracy and better performance.

Fig. 5 shows the STM32F429 Discovery Board, Which is having features like:

1) $180 \mathrm{MHz}$ High speed clock; which helps in feature extraction, digitizing and displaying signals in real time.

2) 2.4" color LCD with $240 \mathrm{RGB} \times 320$ Resolution and also supports touch interface; which helps in displaying the signal as well as the health status of the human body. And touch interface makes it a user friendly.

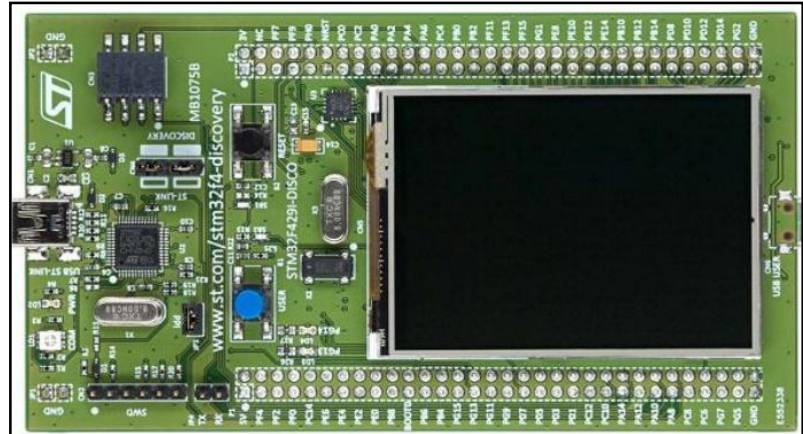

Figure 5: STM32F429I Discovery Board ${ }^{[6]}$.

Differential output of pressure sensor is applied to one of the ADC (A-D Converter) pins of STM32F429 microcontroller for digitization, after amplification. The digitized values are mapped to get them displayed on graphic color LCD of STM32F429I Discovery Board. This digitized values of time domain signal is converted into frequency domain after using the Fast Fourier Transform (FFT). After feature extraction and successful classification, results will be displayed on the LCD as shown in fig. $9 \& 10$.

\section{FEATURE EXTRACTION}

Different methods for extracting features from the wrist pulse signal are:

1) Time domain feature extraction, 2) Time-frequency feature extraction, 3) The curve fitting method ${ }^{[8]}$, 4) The dimension reduction technique (DRT), 5) Frequency domain feature extraction $^{[4][5]}$

Disadvantages of these methods are: Some researchers extracts features from time domain signal and allot these features direct to the classifier for further classification, but sometimes tidal wave and dicrotic wave is very weak in time domain signal, these waves contains much more information regarding the status of the human health. So, one can't diagnose accurately on the basis of time domain signal. Fig. 4 shows the single period time domain signal. Without considering the time complexity, the time-frequency feature extraction is feasible and can obtain good recognition results. The curve fitting may over-fit the data. The key idea of Dimension Reduction Technique (DRT) is to represent the high-dimensional raw data on an intrinsic low dimensional space, but it works with more complexity ${ }^{[4]}$.

In this work, certain features are extracted from the frequency domain wrist pulse. Fast Fourier transform (FFT) is used for converting time domain signal into frequency domain. FFT can be derived from DFT as described below:

We can calculate the Discrete Fourier Transform (DFT) which defined as:

$$
\begin{aligned}
& \mathrm{X}[\mathrm{k}]=\sum_{n=0}^{N-1} \mathrm{x}[\mathrm{n}] * \mathrm{~W}_{\mathrm{N}}^{\mathrm{kn}} \\
& \text { Where } \mathrm{x}[\mathrm{n}]=\text { discrete-time signal } \\
& \mathrm{X}[\mathrm{k}]=\text { frequency domain components } \\
& \mathrm{N}=\text { Number of Points } \\
& \mathrm{k}=0,1,2, \ldots, \mathrm{N}-1
\end{aligned}
$$

Where $\mathrm{W}_{\mathrm{N}}$ in DFT is defined as:

$$
W_{N}=e^{-j\left(\frac{2 \pi n}{N}\right)}
$$


The fast Fourier transform (FFT) reduces the number of calculations of the DFT by dividing the initial function into repeated sub functions and continues this process until the sub function is no longer divisible.

The decimation of the DFT algorithm is defined as:

$$
\begin{aligned}
x[k]=( & \left.\sum_{\mathrm{n}=0}^{\frac{\mathrm{N}}{2}-1}\left(\mathrm{x}[\mathrm{n}]+\mathrm{x}\left[\mathrm{n}+\frac{\mathrm{N}}{2}\right]\right) * \mathrm{~W}_{\frac{\mathrm{N}}{2}}^{\mathrm{kn}}\right) \\
& +\left(\sum_{\mathrm{n}=0}^{\frac{\mathrm{N}}{2}-1}\left(\mathrm{x}[\mathrm{n}]-\mathrm{x}\left[\mathrm{n}+\frac{\mathrm{N}}{2}\right]\right) * \mathrm{~W}_{\frac{\mathrm{N}}{2}}^{\mathrm{kn}}\right)
\end{aligned}
$$

From the calculated FFT Power spectrum of $x[k]$ can be achieved though $\left.\mathrm{PSD}=\operatorname{abs}(\mathrm{fft}(\mathrm{x}[\mathrm{n}]))^{\wedge} 2\right)$.

From this frequency domain features, Band Energy Ratio (BER) is going to be calculated for further processing.

Since, wrist pulse is very low frequency signal, it ranges from $0 \mathrm{~Hz}$ to $20 \mathrm{~Hz}$. Possibility to be energy beyond $20 \mathrm{~Hz}$ is less than $1 \%$ which is negligible. To analyse the energy distribution in the range of $0 \mathrm{~Hz}$ to $20 \mathrm{~Hz}$, the signal is further sub divided into $10 \mathrm{sub}$ bands, each of $2 \mathrm{~Hz}$. Percentage energy in each band is derived from Band Energy Ratio BER (i). Equation for how to calculate BER of each band is shown in equ.5, where ' $i$ ' refers to the band number ${ }^{[5]}$.

$$
B E R(i)=\frac{\text { Energy present in ith band }}{\text { Total Energy within } 0 \text { to } 20 \mathrm{~Hz}} * 100
$$

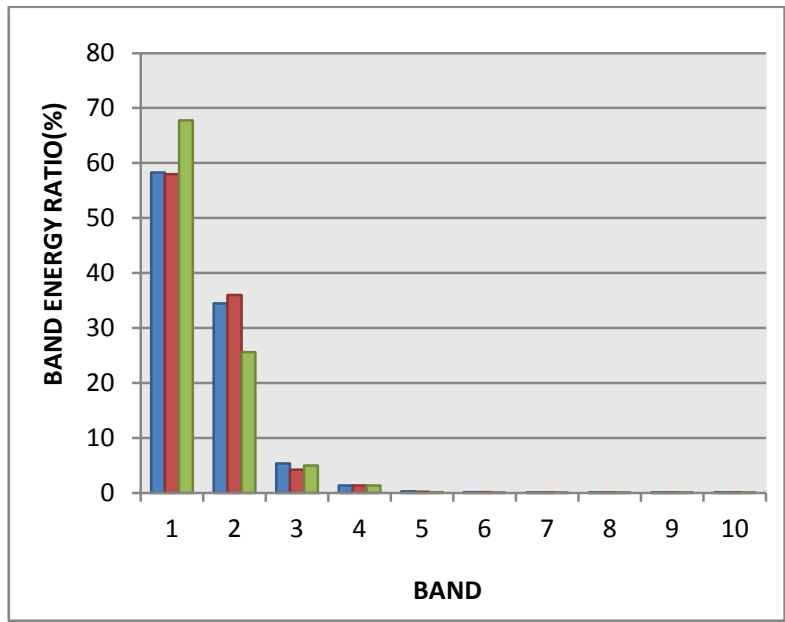

Figure 6: Band Energy Ratio (BER) for three healthy subjects.

Band Energy Ratio (BER) for three healthy and three unhealthy subjects are shown in the fig $6 \& 7$. From figures 6 and 7 one can easily investigate that energy ratio between bands 3 to 5 differs vastly in healthy and unhealthy subjects. Fig. 8 refers to the comparison of energy ratio of bands 3 to 5 of both the subjects. $\mathrm{N}$ stands for normal/healthy subjects and AN for abnormal/unhealthy subject.

From the calculation of the BER, one can get 10 feature elements of the frequency domain signal which together forms a feature vector, this feature vector of BER is given to the equation of binary (linear as well as quadratic) classifier for further classification. How classifier coefficients are to be generated and used in the form of an equation is discussed in the next section.

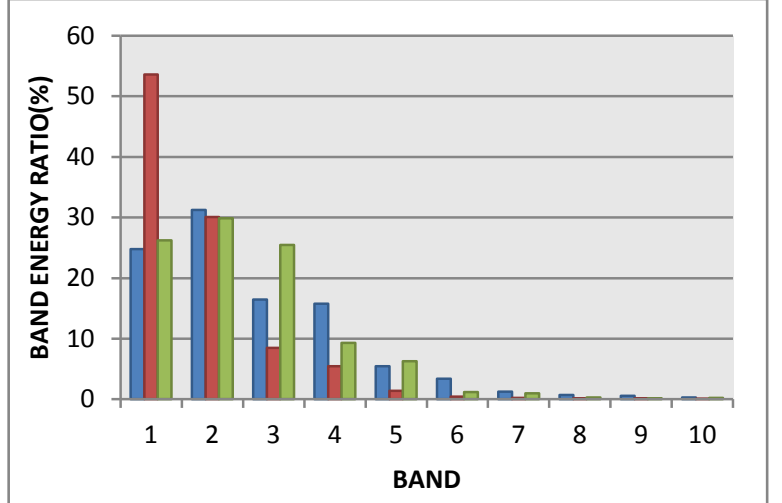

Figure 7: Band Energy Ratio (BER) for three unhealthy subjects.

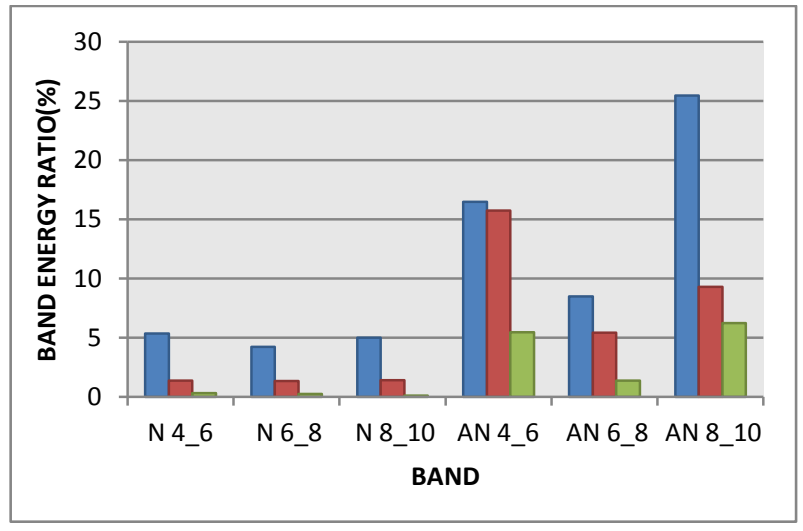

Figure 8: Comparison of bands 3 to 5 between healthy and unhealthy subjects.

\section{CLASSIFICATION}

In this work, linear as well as quadratic classifier is used to classify the health status in a group of two as healthy or unhealthy. A linear classifier accomplishes this by making a classification decision based on the value of a linear combination of the characteristics ${ }^{[7]}$. These characteristics are also known as feature values and are presented in a vector called a feature vector which is given as an input to the classifier. Here the vector of Band Energy Ratio (BER) is provided as an input. For a classification of human health status as healthy or unhealthy, one can visualize the function of linear classifier as separating input data with a hyper plane. The general equation of this hyper plane is same like the line equation as equation 5 .

$$
\begin{aligned}
\mathrm{Y}=\mathrm{a} * \mathrm{x} 1+\mathrm{b}^{*} \mathrm{x} 2+\mathrm{c} & \\
\text { Where, } \mathrm{a}, \mathrm{b} & =\text { linear coefficients. } \\
\mathrm{c} & =\text { constant coefficient. } \\
\mathrm{x} 1, \mathrm{x} 2 & =\text { input data. }
\end{aligned}
$$

The function of quadratic classifier can be visualized as it will separate the data by a conic section (i.e. either a line or a circle or an ellipse etc.). The general equation for quadratic classifier is as discussed in equation 6 .

$$
\mathrm{Y}=\mathrm{a} * \mathrm{x}^{2}+\mathrm{b} * \mathrm{x}+\mathrm{c}
$$

Where $\mathrm{a}=$ quadratic coefficient, $\mathrm{b}=$ linear coefficient, $\mathrm{c}=$ constant coefficient, $\mathrm{x}=$ input data. 
Here the output of Band Energy Ratio (BER) is given as an input feature vector. This vector has 10 feature values as the low frequency band of 0 to $20 \mathrm{~Hz}$ is divided in a sub bands, each of $2 \mathrm{~Hz}$. This will generate 10 linear coefficients and 1 constant coefficient by using the linear classifier and by using diagonal quadratic classifier, it will generate 10 linear coefficients, 10 quadratic coefficients and 1 constant coefficient. For generating the equivalent coefficients for both the classifier, classify command in MATLAB is used. This command will train the classifier according to the groups of training data and classify the testing data according to the same.

$\mathrm{K}$-fold cross validation approach is used to maximize the reliability of the extracted features

In K-fold cross validation, the fundamental samples are randomly separated into $\mathrm{K}$ sub-samples. From these $\mathrm{K}$ sub samples, only single sample is selected as the validation data for testing the model and remaining $\mathrm{K}-1$ sample's are utilized as a training data. These training data trains the classifier in such a way that classifier will classify the testing data into one of the group of the training data. This method repeats for $\mathrm{K}$ times with each of the sample utilized exactly once. Then the $\mathrm{K}$ results from the fold can be averaged to produce single estimation. In our case $\mathrm{K}$ is equal to 10 .

This estimation will provide linear coefficients for linear classifier and linear as well as quadratic coefficients for quadratic classifier.

These coefficients forms an equation of linear plane from linear coefficients of linear classifier and also it will form an equation of conic section from linear and quadratic coefficients of quadratic classifier.

Cortex M4 supports single precision FPU. To use this FPU for better accuracy of the classification, one can use MATLAB's generated double precision coefficients direct to the cortex M4 with modification of placing " $\mathrm{f}$ " after the coefficient.

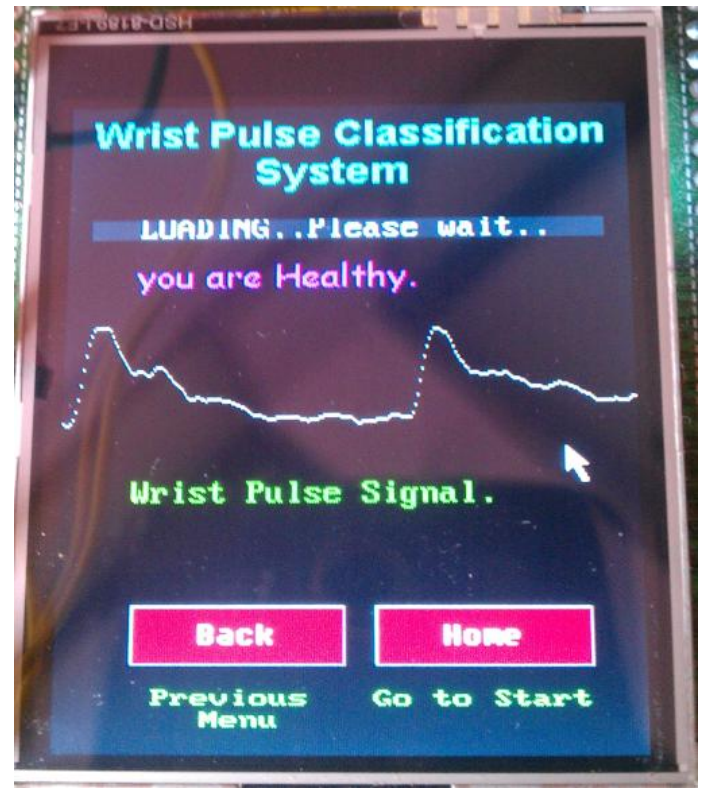

Figure 9: Result for healthy subject after successful classification.

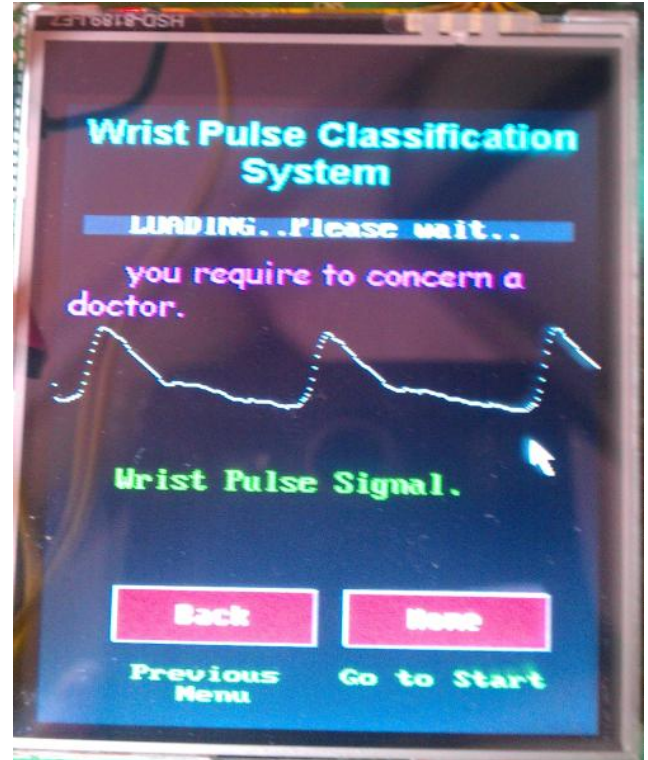

Figure 10: Result for unhealthy subject after successful classification.

Extracted features from the pulse situated in these equations and according to the result one can get the health status. If the result of the equations is positive or zero than the subject is healthy and if the result is negative, the subject is unhealthy. According to these equations health status is carried out.

Result after successful classification is as shown in figures 9 and 10

\section{CONCLUSION}

Radial pulse plays most important part in successful diagnosis of health status. It is observed that wrist pulse is a very low frequency signal and contains much more information about human health status in the frequency band 0 to $20 \mathrm{~Hz}$. For normal subjects energy is quite high in the band 0 to $4 \mathrm{~Hz}$ than rest of the band. For abnormal subjects energy varies vastly in the band 4 to $10 \mathrm{~Hz}$. These extracted features of the signal named as BER is given to the binary (linear as well as quadratic) classifier. This type of energy distribution helps in successful classification of the subjects in a group of two.

For further extension of this work, one can use multiclass classifier for better classification of the human health status. One can also create an android application for continuous monitoring to the remote location.

\section{ACKNOWLEDGEMENT}

We are thankful to Electronics and Communication department of G. H. Patel College of Engineering and Technology, V. V. Nagar, India for providing Lab facilities during this work.

\section{REFERENCES}

[1] Basics of Ayurveda : http://www.quora.com/Why-ispulse-generally-measured-via-the-wrist-or-the-neckrather-than-the-chest-over-the-heart-itself

[2] Traditional method of diagnosis based on wrist pulse. http://livingayurveda.co.uk/pulse-diagnosis-readingwhole-person-pulse/

[3] Guangming Lu, Zhixing Jiang, Liying Ye "Pulse Feature Extraction based on Improved Gaussian Model" 2014 International Conference on Medical Biometrics 
[4] Shujie Gong, Bin $\mathrm{Xu}$, Guodong Sun, Mingrui Chen,Nanyue Wang, Chiyu Dong, Peng Wang, Jian Cui," Accurate Cirrhosis Identification with Wrist-Pulse Data for Mobile Healthcare", mHealthSys 2012 Toronto, Canada

[5] Bhaskar Thakker, Anoop L. Vyas," Frequency Domain Analysis of Radial Pulse in Abnormal Health Conditions", 2010 IEEE EMBS Conference on Biomedical Engineering \& Sciences (IECBES 2010), Kuala Lumpur, Malaysia, 30th November - 2nd December 2010.

[6] Datasheet of STM32F429xx

[7] Basics of linear classifier https://en.wikipedia.org/wiki/Linear_classifier
[8] Complex Fixed-Point Fast Fourier Transform Optimization for AltiVec ${ }^{\mathrm{TM}}$, Freescale Semiconductor Application Note, Document Number: AN2114Rev. 4, $04 / 2013$

[9] Bhaskar Thakkar , Anoop Lal Vyas, "Suppressed Dicrotic Notch Pulse Classifier Design" International Journal of Machine Learning and Computing, Vol. 1, No. 2, June 2011

[10] Bhaskar Thakker and Anoop Lal Vyas, "Support Vector Machine for Abnormal Pulse Classification" International Journal of Computer Applications 22 (7):13-19, May 2011 